\title{
Numerical studies of the effects of the fluid density on the flow structures in circulating fluidized beds
}

\author{
Zeneng Sun $^{1}$, Chao Zhang ${ }^{2 *}$, Jesse Zhu ${ }^{1}$ \\ ${ }^{1}$ Department of Chemical and Biochemical Engineering, Western University, London, Canada \\ ${ }^{2}$ Department of Mechanical and Materials Engineering, Western University, London, Canada \\ *Corresponding author e-mail address: czhang@eng.uwo.ca
}

\begin{abstract}
The effects of the fluid density on the flow structures in the circulating fluidized bed (CFB) are numerically studied. The studies of a benchmark gas-solid CFB case and 3 other cases with the fluid density of 500, 1000 , and $1200 \mathrm{~kg} / \mathrm{m}^{3}$ are carried out under the equivalent operating conditions. The axial and radial profiles of the average phase holdups and their corresponding standard deviations are obtained and compared. The average velocity profiles and the corresponding standard deviations of the fluid and solid phases in $\mathrm{X}$ and $\mathrm{Y}$ directions are also provided. Results show that a smaller density difference between the fluid and solid phases leads to a less fluctuating flow structures in the CFB reactor.
\end{abstract}

Keywords-circulating fluidized bed; fluid density; flow structure

\section{INTRODUCTION}

A fluidized bed is a commonly used chemical reactor which applies the fluidization process to handle the granular materials in an easier way with the help of a fluid. By introducing a fluid at a certain flowrate into a column with packed particles, the solid particles will be suspended and can behave like liquid since the drag force from the passing fluid on the particles is large enough to balance the net weight of the particles [1]. With the increase in the fluid velocity, the particles might be entrained out of the column due to the greater drag force. A circulating fluidized bed (CFB) can be developed by collecting and recycling the entrained particles back to the column [2]. The CFB has been firstly commercialized since the late 1940s and then widely applied into the industries including oil refinery processes, coal gasification and combustion, and particle transport processes [2].

Either gas or liquid can work as the fluidizing agent and can result in different flow regimes in the fluidized beds due to the density or viscosity differences between the solid and fluid. Liquid-solids fluidization system is usually more homogeneous as known as the particulate fluidization, however, gas-solids fluidization is considered as aggregative fluidization where solids tend to aggregate to form clusters [2]. The density difference between the fluid and solids may play an important role in the transition from a particulate fluidization to an aggregative fluidization. A smaller density difference such as liquid-solids fluidization causes less shear force between the fluid and particles in a fluidized bed, so that particulate fluidization is more likely to happen. Under particulate fluidization, liquid-solids flow structure is generally uniform with several basic flow regimes. However, larger density difference, such as gas-solids fluidization, increases the shear force and contributes to aggregation. Under aggregative fluidization, hydrodynamics is more complicated resulting in various flow regimes with different flow structures.

Currently, the flow regime mapping work was done separately on either the liquid-solids fluidization system or gassolids fluidization system. However, how the transition from particulate fluidization to aggregative fluidization takes place is still unknown. By adjusting the fluid density, it is expected that a closer density difference between fluid and particles helps keep particles flow with the fluid inside the fluidized bed. Consequently, the homogeneity of the fluidization system can be improved with a reduction of the shear force between the fluid and particles. However, there existing a gap in the fundamental research study of how the fluid density effects on the fluidization flow structures, because of the limitation of the experimental techniques and the lack of the certain types of fluid with various densities across gas to liquid on the experimental study, and the operability of the experiment is very low. Therefore, computational fluid dynamic (CFD) method becomes an effective tool to numerically study the effects of different fluids to the transitions between fluidization regimes.

The CFD approach has been widely applied into the studies of CFBs since the 1970s and achieved considerable success on predicting the flow structures of the multiphase flow in the CFB with more accurate mathematical descriptions and more realistic assumptions recently [3]. Both the simulations of the liquid-solid and gas-solid CFB systems have achieved good agreements with the experimental results, which indicates that the CFD model for simulations of CFBs has been well validated [4]. Additionally, with the help of CFD, a wider operating range of the CFBs can be numerically studied because the properties of the fluid and particles can be adjusted freely. In this work, the Eulerian-Eulerian two-fluid model 
(EE-TFM) is selected to study the effects of the fluidizing agent on the CFBs. Based on the gas-solid and liquid-solid CFB systems, the CFD-TFM simulations on CFBs where the fluid phase with different densities were conducted to give some insights into the flow regime transition from liquid-solid system to the gas-solid system.

\section{COMPUTATIONAL DOMAIN AND MESH INFORMATION}

The computational domain used in this work is based on an upward CFB reactor of $10 \mathrm{~m}$ high and $0.2 \mathrm{~m}$ in diameter as shown in Fig. 1. Both the fluid and particles enter the CFB from the bottom with an upward velocity. The inlets of the fluid and solid phases located at the bottom and the outlets of the both phases located at the top of the computational domain. A 2D quad grid system with finer mesh at the entrance and the wall region is developed as shown in Fig. 2. The mesh consists of a total 12,000 grids with 60 nodes in the radial direction and 2,000 nodes in the axial direction. The maximum aspect ratio of the mesh is 5.94. The grid independent test was completed in the previous study.

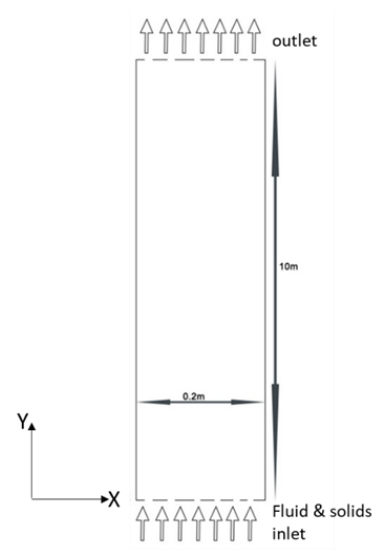

Figure 1. Schematic of the circulating fluidized bed reactor

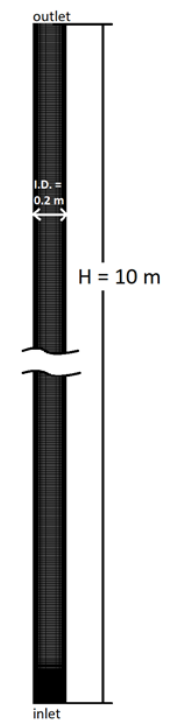

Figure 2. 2D mesh of the computational domain

\section{CFD MODEL DESCRIPTIONS}

\section{A. EE-TFM method for CFB reactor}

The EE-TFM CFD model coupling with the kinetic theory of granular flow (KTGF) for the solid phase is used in this work. The governing equations and closure equations of the EE-TFM are listed in Tab.1. In EE-TFM, both the fluid and solid phases are treated as interpenetrating continua. A set of governing equations of the continuity and momentum of each phase are discretized and solved by the EE-TFM. The pressure and viscosity of the solid phase are derived from the granular temperature of the solid particles which associates with the fluctuations of the particles in the KTGF model. The standard $\mathrm{k}-\varepsilon$ turbulence model is selected for both the fluid and solid phases because it is reported to be robust in the simulation of CFBs. The interactions between the fluid and particles are concluded in the calculation of drag by the drag model. The commonly used Syamlal-O'Brien drag model based on the R-Z equation and particle terminal velocity is selected in this work.

The commercial software Ansys Fluent 17.0 is used for the simulation. A pressure-based solver with the SIMPLE scheme is selected. Second order upwind discretization scheme is selected for the momentum equation, turbulent model, and the granular temperature schemes. The residual criteria for each scheme were set as 0.0005 . The time step size was set as $0.0001 \mathrm{~s}$ at first and adjusted to $0.001 \mathrm{~s}$ after the simulation is closed to the steady state.

\section{B. CFD cases setup and boundary conditions}

Since the purpose of this work is to study the relationship between the fluid densities and the fluidization flow structures process, multiple types of fluids with different densities will be considered in the simulations. Firstly, the selection of the operating condition is based on a gas-solid CFB system, which can be considered as the benchmark operating condition. Secondly, the selection of the fluids is based on two the most commonly seen fluids, air. Based on the benchmark fluid (air), liquid water and other two virtual fluids with densities of 500 $\mathrm{kg} / \mathrm{m}^{3}$ and $1200 \mathrm{~kg} / \mathrm{m}^{3}$ were selected to be tested in this work. The viscosity of these two virtual fluids were obtained from the interpolation of the air and water viscosities, which might not be very appropriate and should be studied in the future. Typical Group A particles with an average diameter of $0.0001 \mathrm{~m}$ and an average density of $1500 \mathrm{~kg} / \mathrm{m}^{3}$ was selected as the solid phase in the simulation.

One challenge in this study is how to link a series of CFD cases with different fluids to the equivalent operating conditions for a fair comparison. A gas-solid CFB case with the superficial gas velocity of $10 \mathrm{~m} / \mathrm{s}$ and the solids circulation rate of $200 \mathrm{~kg} / \mathrm{m}^{2} \mathrm{~s}$ was selected as the benchmark operating condition to establish as the reference for the setup of the other cases. The particle terminal velocity $\left(U_{t}\right)$ which represents the constant velocity of one particle freely falling in the corresponding fluid is introduced in the determination of the operating conditions in the CFB with different types of fluids. Since $U_{t}$ only relates with the particle diameter and the density difference between the fluid and solid phases, it is fair enough to select $U_{t}$ as the parameter to link all the operating conditions. 
The calculation of $U_{t}$ in fluidized bed is from the force balance of the particle:

$$
U_{t}=\sqrt{\frac{\sqrt{G \epsilon_{p}}}{a C_{d I}}\left(\frac{\rho_{x}-\rho_{f}}{\rho_{f}}\right)}
$$

where $d_{p}$ is the diameter of the particles, $\rho_{f}$ and $\rho_{s}$ are the are the densities of the fluid and particles, $C_{d}$ is the drag coefficient of particles.

The basic assumption to link the CFD cases with different fluid densities with the benchmark case under the equivalent operating conditions is that the ratio of the particle terminal velocities in the fluid to be tested (T-fluid) and the benchmark fluid (B-fluid) should be the same as the ratio of the testing fluid and the benchmark fluid velocities, as shown below.

$$
\frac{U_{B-\text { fluid }}}{U_{t_{B}-\text { fluid }}}=\frac{U_{T-\text { fluid }}}{U_{t_{T}-\text { fluid }}}
$$

Therefore,

$$
\frac{G_{S_{B}-\text { fluid }}}{U_{t_{B}-\text { fluid }}}=\frac{G_{S} T \text {-fluid }}{U_{t_{-} T-\text { fluid }}}
$$

Where $U t_{B-f l u i d}$ and $U t_{T-\text { fluid }}$ are the particle terminal velocities in the benchmark case (air) and the fluid to be tested, respectively, $U_{B-\text { fluid }}$ and $U_{T-\text { fluid }}$ are the superficial velocities of the benchmark fluid and testing fluid, respectively, $G s_{B-f l u i d}$ and $G s_{T-\text { fluid }}$ are the solids circulation rates for the benchmark fluid case and the testing fluid case, respectively. Thus, the superficial fluid and solid velocities for the testing cases can be determined based on Eqs. (2) and (3).

In the simulations, the inlet for both the fluid and solid phases are located at the bottom and the outlet located at the top of the CFB as shown in Fig. 2. Uniform velocity inlet boundary condition is applied to both the fluid and solid phases. A volume fraction $(\varphi)$ of 0.3 is selected for the solid phase at the inlet since the solid suspension flow is quite dense at the inlet. So that, the inlet velocities of the fluid and particles can be obtained from the superficial fluid velocity and the solid circulation rate:

$$
\begin{gathered}
V_{f_{-} \text {in }}=\frac{U_{\text {fluid }}}{1-\varphi} \\
V_{s_{-} \text {in }}=\frac{G_{g \text { fluid }}}{\rho_{g^{-} \varphi}}
\end{gathered}
$$

The Johnson-Jackson wall boundary condition with a restitution coefficient of 0.9 and a specularity coefficient of 0.0001 is applied in the simulations. The simulations for the

\begin{tabular}{|c|c|c|c|c|}
\hline & $\begin{array}{c}\text { Benchmark } \\
\text { Case } \\
\text { (gas-solid) }\end{array}$ & Case 1 & $\begin{array}{c}\text { Case 2 } \\
\text { (liquid- } \\
\text { solid) }\end{array}$ & Case 3 \\
\hline Fluid density $\left(\mathrm{kg} / \mathrm{m}^{3}\right)$ & 1.22 & 500 & 1000 & 1200 \\
\hline $\begin{array}{c}\text { Viscosity of fluid, } \\
\text { (Pa.s) }\end{array}$ & 0.000018 & 0.0005242 & 0.001003 & 0.001194 \\
\hline Particle diameter $(\mathrm{m})$ & 0.0001 & 0.0001 & 0.0001 & 0.0001 \\
\hline $\begin{array}{l}\text { Particle density } \\
\qquad\left(\mathrm{kg} / \mathrm{m}^{3}\right)\end{array}$ & 1500 & 1500 & 1500 & 1500 \\
\hline $\begin{array}{l}\text { Particle terminal } \\
\text { velocity, } U t(\mathrm{~m} / \mathrm{s})\end{array}$ & 0.365 & 0.00903 & 0.00255 & 0.001306 \\
\hline $\begin{array}{c}\text { Superficial fluid } \\
\text { velocity, } U_{\text {fluid }},(\mathrm{m} / \mathrm{s})\end{array}$ & 10 & 0.266 & 0.07 & 0.0357 \\
\hline $\begin{array}{l}\text { Inlet fluid velocity, } \\
\qquad V_{f_{i} \text { in }}(\mathrm{m} / \mathrm{s})\end{array}$ & 14 & 0.38 & 0.10 & 0.051 \\
\hline $\begin{array}{l}\text { Solids circulation rate, } \\
\text { Gs }\left(\mathrm{kg} / \mathrm{m}^{2} \mathrm{~s}\right)\end{array}$ & 198 & 4.95 & 1.395 & 0.07 \\
\hline $\begin{array}{l}\text { Inlet solid velocity, } \\
\qquad V_{s_{-} i n}(\mathrm{~m} / \mathrm{s})\end{array}$ & 0.44 & 0.011 & 0.0031 & 0.0016 \\
\hline $\begin{array}{l}\text { Particle-wall } \\
\text { restitution coefficient }\end{array}$ & 0.9 & 0.9 & 0.9 & 0.9 \\
\hline $\begin{array}{c}\text { Specularity coefficient } \\
\text { at wall }\end{array}$ & 0.0001 & 0.0001 & 0.0001 & 0.0001 \\
\hline
\end{tabular}
benchmark case and additional three cases with different fluid densities were carried out in this work. All the operating conditions and boundary conditions are listed in Table 1.
TABLE I. OPERATING CONDITIONS AND BOUNDARY CONDITIONS

IV. RESULTS AND DISCUSSION

\section{A. Axial solids holdup distributions}

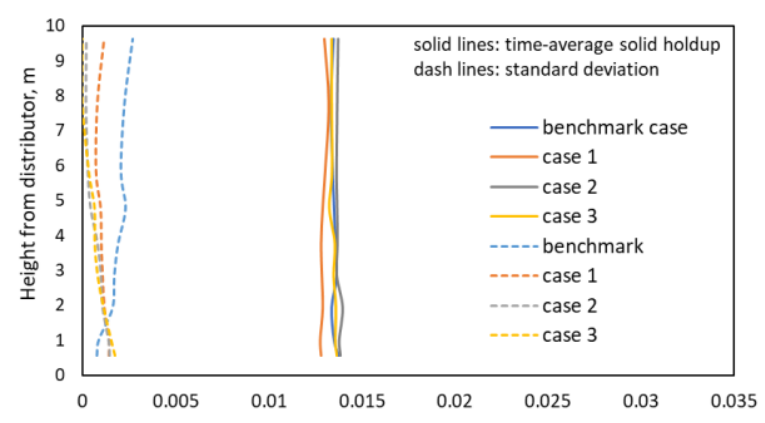

Figure 3. Time-averaged cross-sectional solids holdup and standard deviation

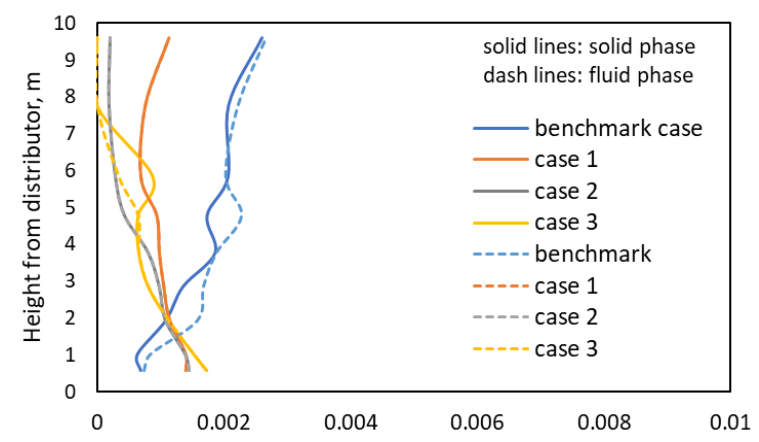

Figure 4. Comparison of the standard deviations of the volume fraction for fluid and solid phases

The average cross-sectional solid holdups and its standard deviations of each cases are plotted as shown in Fig. 3. It can be found that the fluid density has little effect on the solid holdup as well as the axial distribution of the solid holdup. The 
average solid holdups of all the 4 cases are very close to 0.013 although the fluid density changes from 1.22 to $1200 \mathrm{~kg} / \mathrm{m} 3$. The axial distributions of the average solids holdup are also very uniform along the entire CFB, so that the corresponding standard deviations are also small for all the cases. However, the standard deviations of the solid holdups for each case are not as close as the average solid holdup as shown in Fig.3. A comparison of the standard deviations of the phase holdups between the fluid and solid phases are plotted as shown in Fig. 4. It is clear that the standard deviation of the fluid phase holdup is close to the solid phase. The benchmark case of which air is used as the fluid phase has the highest standard deviations of the phase holdup since the density difference between air and the solid is the largest among the four cases resulting in greater fluctuations of the system. With the increase in the fluid density, the standard deviations of the phase holdup decreases as shown in Fig. 4, which indicates that a smaller density difference between the fluid and solid results in lower fluctuations of the system. Another interesting finding is that the standard deviation of the phase holdup increases from the bottom to the top of the CFB when the density difference between the fluid and particles is large such as the benchmark case, and in turn decreases along the axial direction when the density difference is small. This phenomenon might indicate that the density difference between the fluid and the particles has some impacts on the development of the multiphase flow in the CFB reactor, and a smaller density difference results in a more stable and faster development of the flow because the slip velocity between the fluid and particles is smaller.

\section{B. Radial solids holdup distributions}

Time-averaged radial solid holdup profiles at different heights along the CFB for each case are compared as shown in Fig. 5. Generally, the radial distributions of the solid holdup of all of the 4 cases are relatively uniform around 0.013 at different heights along the CFB reactor as shown in Fig. 5. Compared with the other three cases, the benchmark case has some fluctuations of the radial solid holdup at the bottom $(\mathrm{h}=$ $1.94 \mathrm{~m})$ and middle $(\mathrm{h}=4.78 \mathrm{~m})$ zones of the CFB reactor. As discussed before, since the density difference between the fluid and solid is the largest for the benchmark case, more intensive interactions between the gas and particles are excepted with a higher slip velocity resulting in a longer development region of the multiphase flow with more fluctuations. In the fully developed region $(\mathrm{h}=9.61 \mathrm{~m})$ of the CFB reactor, all of the 4 cases achieve a uniform radial distribution of the solid holdup.

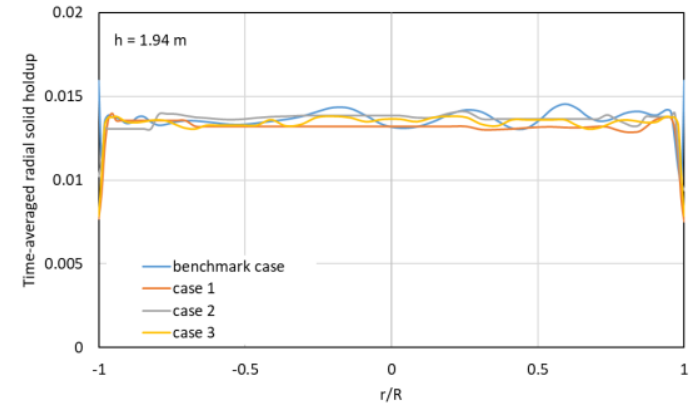

(a) $\mathrm{h}=1.94 \mathrm{~m}$

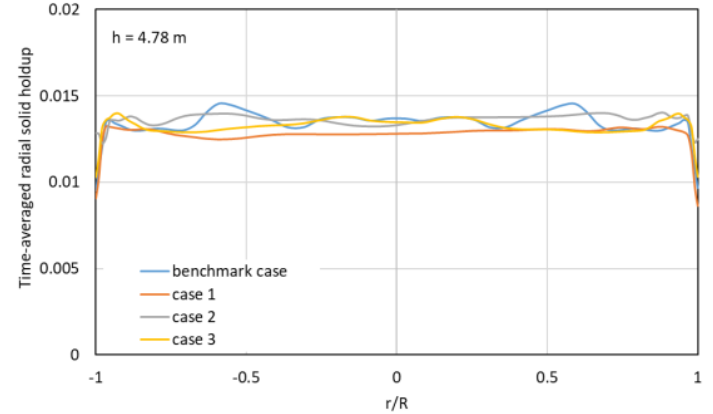

(b) $\mathrm{h}=4.78 \mathrm{~m}$

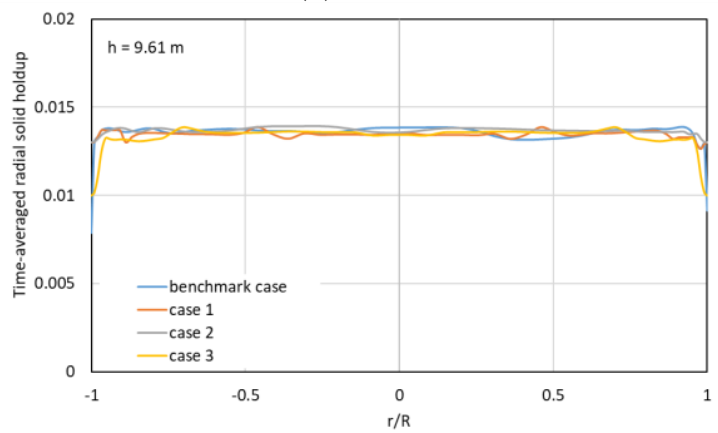

(c) $\mathrm{h}=9.61 \mathrm{~m}$

Figure 5. Comparison of time-averaged radial distributions of the solid holdup at different heights

At this point, combining with the axial and radial solid holdup distributions, a conclusion can be drawn that: by changing the fluid density, the overall bed density (average solid holdup in the CFB) keeps constant under the equivalent operating conditions $\left(\frac{U_{B-\text { fluid }}}{v_{t_{T} \text {-fluid }}}=\frac{U_{T-\text { fluid }}}{v_{t_{T} \text {-fluid }}}=\frac{G_{S_{B-\text { fluid }}}}{G_{S_{T-\text { fluid }}}}\right.$ ). In another word, the fluid density does not have a distinct impact on the overall bed density if the operating fluid velocity and the solid circulation rate are set based on the ratio of the particle terminal velocities between the benchmark system and other systems, i.e.

$$
\bar{\varepsilon}_{s}=\bar{\varepsilon}_{s \text { benchmark }}
$$

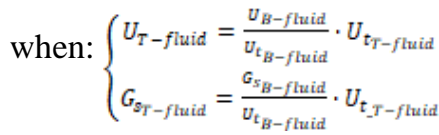

\section{Distributions of fluid and solid velocities}

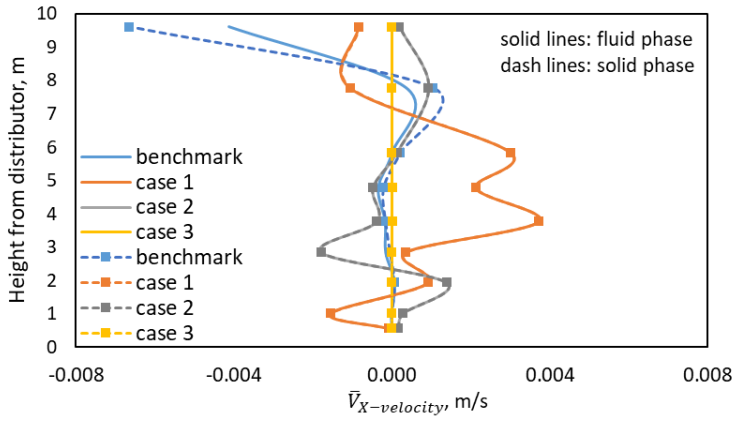

(a) X-velocity profiles 


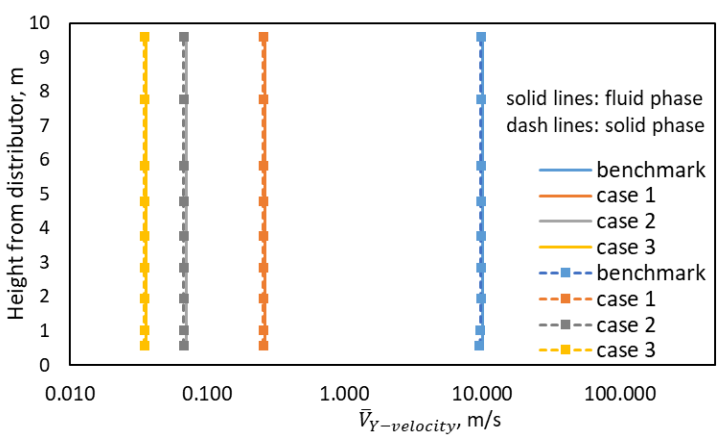

(b) Y-velocity profiles

Figure 6. Comparisons of average $\mathrm{X}$ and $\mathrm{Y}$-velocities for fluid and solid phases

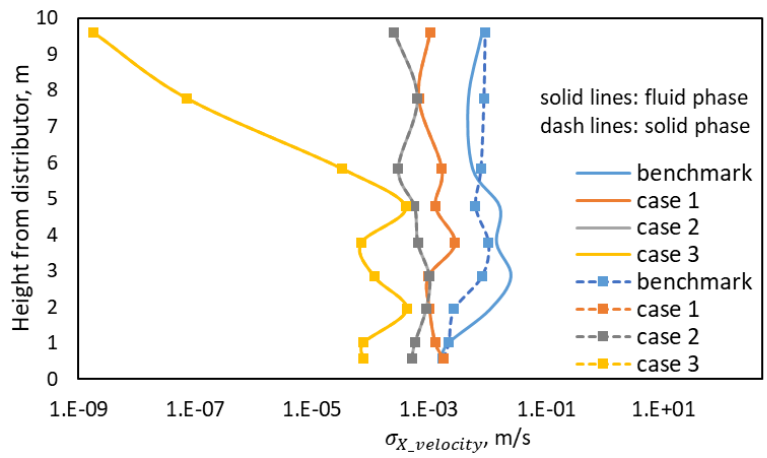

(a) Standard deviation profiles of X-velocity

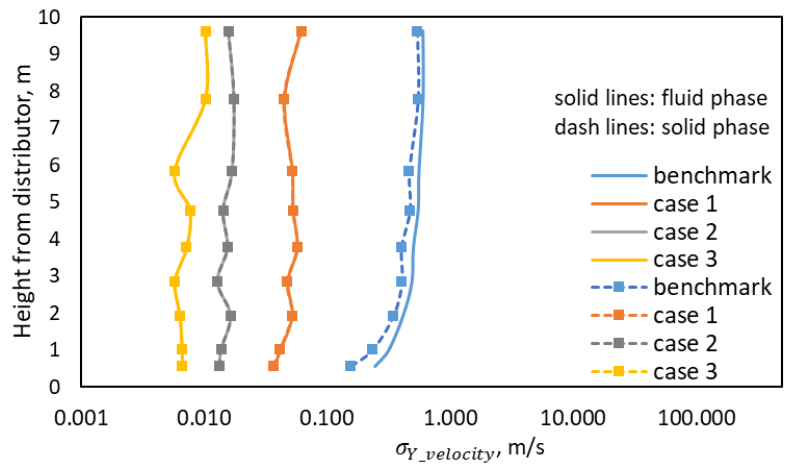

(b) Standard deviation profiles of Y-velocity profiles

Figure 7. Comparison of the standard deviations of $\mathrm{X}$ and $\mathrm{Y}$-velocity for fluid and solid phases

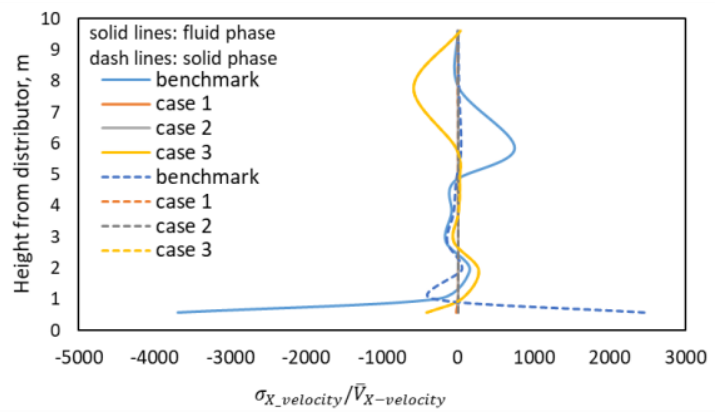

(a)

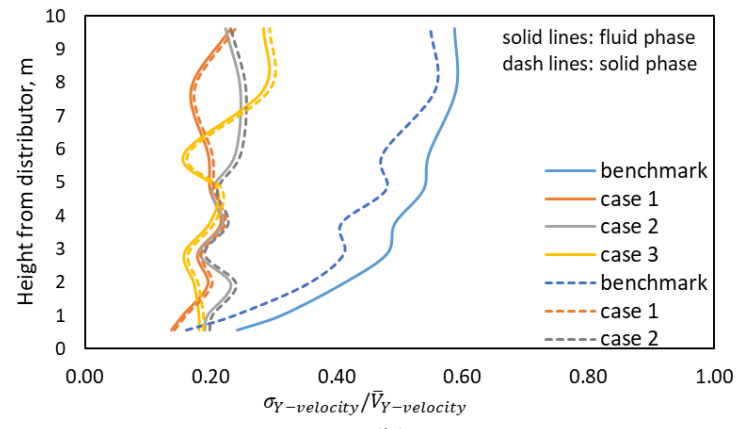

(b)

Figure 8. Comparison of the relative standard deviations of X and Y-velocity for fluid and solid phases

The average lateral velocity $(\mathrm{X})$ and axial velocity $(\mathrm{Y})$ of the fluid and solid phases along the CFB reactor are compared as shown in Fig. 6. Apparently, the Y-velocities between the fluid and solid phases are almost the same and quite uniform for each case as shown in Fig. 6 (b). The X-velocities for both the fluid phase and the solid phase are also very close except for the benchmark case which has the largest density difference. However, the $\mathrm{X}$-velocities have less uniform distributions along the CFB except for case 3 although the Xvelocities are smaller than the Y-velocities as shown in Fig. 6 (a). With a smaller density difference, less fluctuations are found in the X-velocity profiles as shown in Fig. 6 (a) due to the lower slip velocity. More details can be found in the profiles of the corresponding standard deviations of the $\mathrm{X}$ and $\mathrm{Y}$ velocities as shown in Fig. 7. Same trend with the average velocity profiles is found in the profiles of their standard

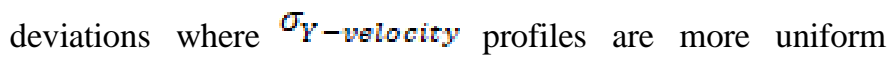
comparing with the $\sigma_{X \text {-velocity }}$ profiles for all the 4 cases as shown in Fig. 7. With the smaller density difference between the two phases, the standard deviations of both the $\mathrm{X}$ and $\mathrm{Y}$ velocity also decrease, which also echoes the standard deviation profiles of the phase holdup (Fig. 4). A smaller

$\sigma_{X \text {-velocity indicates less intensive lateral interactions }}$ between the fluid and particles, and as mentioned above, a smaller density difference results in a smaller slip velocity, so that the particles tend to flow along with the upward flowing fluid. Since the operating velocities are different in each case, the relative standard deviation of the velocity which is the ratio of the standard deviation and the average velocity in $\mathrm{X}$ and $\mathrm{Y}$ directions are shown in Fig. 8 for a fair comparison by eliminating the effect of the magnitude of the velocity. It can be found that the relative standard deviation in the $\mathrm{X}$ direction $\left(\sigma_{X \text {-velocity }} / \bar{V}_{X \text {-velocity }}\right)$ is larger than that in the $\mathrm{Y}$ direction $\left(\sigma_{Y \text {-velocity }} / \bar{V}_{Y \text {-velocity }}\right)$ as shown in Fig, 8 , which further proves that more intensive lateral interactions occur in the benchmark case than other cases as show in Fig. 8 (b).Therefore, it can be concluded that a larger density difference between the two phases results in more intensive interactions between the fluid and solid, so that a more aggregative fluidization could take place. 


\section{CONCLUSIONS}

The effects of the fluid density on the fluidization flow structures in the CFB reactor have been studied numerically in this work. The overall bed density in the CFB keeps constant when varying fluid densities under the equivalent operating

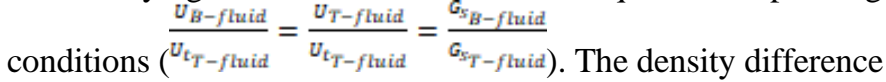
between the two phases has some impacts on the development of the multiphase flow in the CFB reactor. A smaller density difference between the fluid phase and the solid phase results in a less fluctuating flow structures with smaller standard deviations of the solid holdup, and $\mathrm{X}$ and $\mathrm{Y}$ velocities for both the fluid and solid phases. Future studies in a wider operating window are needed for further understanding the fluidization mechanism.

\section{REFERENCES}

[1] Grace, J. R. (1986). Contacting modes and behaviour classification of gas-solid and other two-phase suspensions. The Canadian Journal of Chemical Engineering, 64(3), 353-363.

[2] Grace, J. R., Bi, H., \& Golriz, M. (2003). Circulating fluidized beds. In M. D. Wen-Ching Yang (Ed.), Handbook of fluidization and fluidparticle systems (pp. 485-498). New York: CRC press.

[3] Hartge, Ernst-Ulrich, et al. CFD-simulation of a circulating fluidized bed riser. Particuology 7.4 (2009): 283-296.

[4] Benyahia, S., et al. Simulation of particles and gas flow behavior in the riser section of a circulating fluidized bed using the kinetic theory approach for the particulate phase. Powder Technology 112.1 (2000): 24-33.

[5] ANSYS, I. (2013). ANSYS Fluent Theory Guide. 